\title{
Amiloidosis vesical: una lesión que simula un tumor
}

\section{Bladder amyloidosis: a lesion that simulates a tumor}

\section{Sr. Director:}

La amiloidosis consiste en el depósito extracelular de amiloide, una proteína de estructura fibrilar, en una o más localizaciones del organismo. Fue descrito por vez primera por Virchow en 1853.

El interés clínico del conocimiento de esta patología se fundamenta en el hecho de que la amiloidosis primitiva localizada únicamente en la vejiga, pueda confundirse fácilmente con una neoplasia infiltrante debido a su aspecto ${ }^{1}$.

Mostramos el caso de una paciente de 67 años entre cuyos antecedentes de interés destaca una hipercolesterolemia y espondiloartrosis. Es remitida del ambulatorio por sospecha de tumor vesical, habiendo presentado hematuria macroscópica de 3 días de evolución y síndrome irritativo miccional.

La analítica de orina no presentaba alteraciones significativas y una ecografía abdomino pélvica revelaba riñones de tamaño y morfología normales, sin dilatación de la vía excretora, visualizándose un discreto engrosamiento de la pared vesical. Como consecuencia de ello, se sometió a la paciente a una cistoscopia en la que se observó un tapizado inflamatorio sugestivo de un posible tumor infiltrante alrededor del orificio ureteral izquierdo. La citología urinaria resultó negativa, se intervino a la paciente mediante resección transuretral extirpando en su totalidad dicha región que macroscópicamente ofrecía dudas diagnósticas.

El diagnóstico anatomo patológico no evidenció signos de malignidad, tratándose de un caso de amiloidosis vesical. La pared vesical mostraba en el tejido conectico subepitelial, abundante material extracelular homogéneo y eosinófilo. Y realizada la técnica de Rojo Congo, dicho material mostraba birrefringencia al ser observado con luz polarizada (figs. 1 y 2).

En el caso de nuestra paciente hemos optado por un tratamiento sintomático conservador expectante, realizando

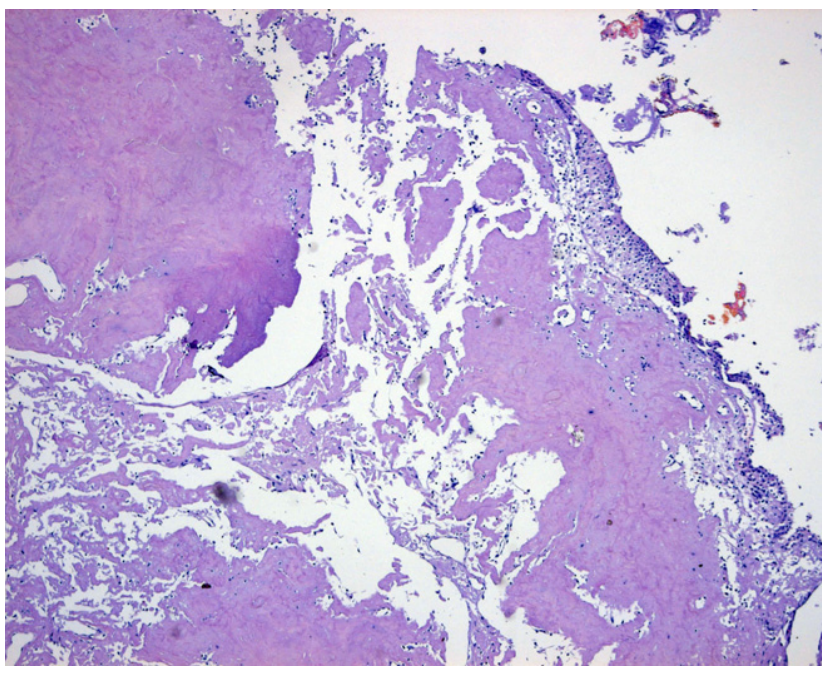

Figura 1 - Imagen anatomo-patológica de amiloidosis vesical. revisiones periódicas con cistoscopias y a pesar de que el tiempo de evolución es corto, no se han presentado recidivas por el momento.

Se pueden clasificar las formas de amiloidosis del tracto genitourinario en dos grupos: formas primarias o idiopáticas y formas secundarias.

La amiloidosis secundaria de la vejiga es la participación de este órgano en un proceso sistémico generalizado, como procesos infecciosos o inflamatorios crónicos, neoplasias, etc., siendo la artritis reumatoide la enfermedad sistémica más frecuentemente asociada a esta entidad ${ }^{2,3}$. En lo que a la lesión vesical se refiere, muestra una evolución tórpida, con aparición de hematurias repentinas de carácter intenso, en ocasiones muy graves y de difícil tratamiento, sin relación con los escasos síntomas percibidos hasta ese momento ${ }^{4,5}$. El factor desencadenante de la hematuria no es del todo bien conocido, aunque se cree que pueda estar en relación con la irritación de la mucosa vesical por factores como infecciones urinarias, instrumentaciones endoscópicas.

Existen ciertas amiloidosis sistémicas generalizadas que surgen en personas sin evidencia de patología causal, sin neoplasias, mielomas..., en las cuales la afectación vesical se caracteriza por ser más difusa, mostrando aspecto de cistitis hemorrágica generalizada y sin lesiones pseudotumorales.

Por otra parte, se encuentra la amiloidosis primitiva, localizada únicamente en la vejiga. Se trata de una cistopatía poco frecuente. Suele aparecer en personas jóvenes, con buena salud aparentemente, con cierta predominancia en el sexo femenino ${ }^{3}$. Debuta con hematurias asintomáticas y los hallazgos cistoscópicos pueden revelar zonas hemorrágicas con áreas de edema alrededor que proporcionan un aspecto de proceso inflamatorio ulcerado o pueden simular un tumor infiltrante, sin embargo en la citología del sedimento urinario

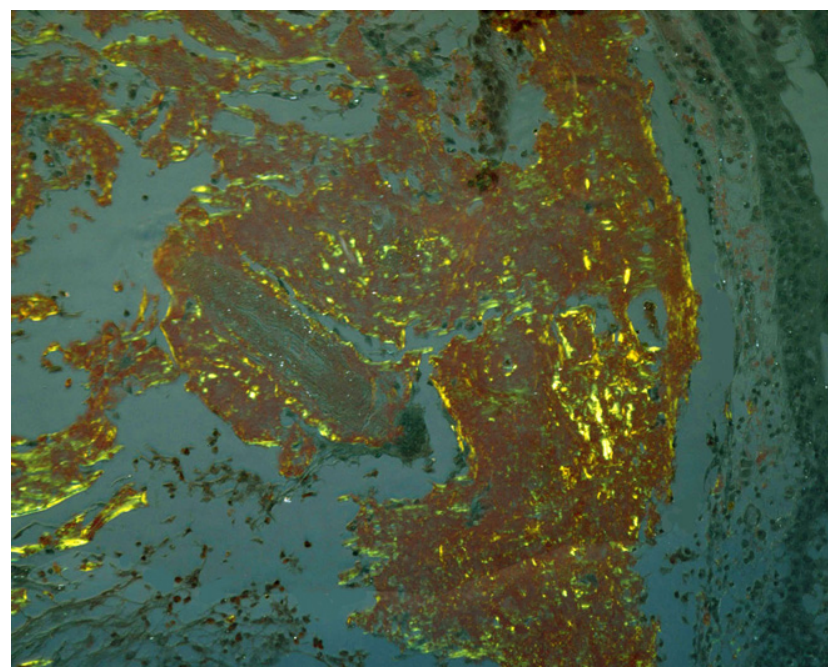

Figura 2 - Tras tinción Rojo Congo. 
no se visualizan células neoplásicas. De ahí que, ante una lesión de aspecto tumoral con citología urinaria negativa, se debe pensar en la posibilidad de que nos encontremos ante un caso de amiloidosis primitiva ${ }^{6}$. La visión endoscópica de placas amarillentas también puede hacernos sospechar la presencia de una amiloidosis. Esta enfermedad vesical primaria puede evolucionar de una forma lenta afectándose progresivamente toda la pared de la vejiga incluyendo los nervios, originando una neuropatía sensitiva que conlleva a un trastorno de vaciado de la vejiga. Así, en etapas tardías de la enfermedad se comporta como una vejiga neurógena. El examen anatomo patológico revelará la ausencia de neoplasia. Los depósitos de amiloide generalmente invaden los vasos y el tejido conectivo submucoso, estando menos frecuentemente afectada la muscular ${ }^{7}$. La sustancia amiloidea teñida con Rojo Congo ${ }^{3}$ adopta birrefringencia verdosa observada con luz polarizada. Si se tiñe con tioflavina $\mathrm{T}$ también presenta birrefringencia. Resulta interesante la tipificación inmunohistoquímica con anticuerpos específicos contra los diferentes componentes del amiloide para el diagnóstico ${ }^{8}$. La mayoría de las formas localizadas contienen, además de la proteína amiloide $\mathrm{A}$, depósitos de cadenas ligeras de inmunoglobulinas monoclonales (de tipo AL). Los depósitos exclusivamente formados por proteína A (de tipo AA) sugieren amiloidosis secundaria.

Otras pruebas útiles para diferenciar la enfermedad primaria de la sistémica son la biopsia de la mucosa rectal y grasa abdominal, pruebas funcionales orgánicas como creatinina, proteinuria, transaminasas, etc. y el ecocardiograma.

En cuanto al tratamiento, se recomienda en estos casos de amiloidosis vesical localizada una resección transuretral amplia, la cual facilita tras la intervención endoscópica un vaciamiento lento de la sustancia amiloide intersticial, lo que ayudará a la curación. Una vez realizada la resección endoscópica, puede resultar útil el tratamiento local con dimetilsulfóxido (DMSO) en instilaciones vesicales de $50 \mathrm{ml}$ al $50 \%$ durante $30 \mathrm{~min}$, repitiéndose cada 15 días $^{9}$.

La colchicina también se emplea como tratamiento oral a largo plazo en caso de amiloidosis a dosis de 0,5 mg una o 2 veces al día ${ }^{1}$. Está descrita la ligadura de las arterias hipogástricas, incluso la cistectomía para casos extremos en los que se ha de recurrir a tratamientos más agresivos ${ }^{3}$.

A su vez, se exige un estudio y un seguimiento prolongado de por vida mediante cistoscopias en estos pacientes debido a la frecuencia de multiples recurrencias ${ }^{9}$ y en ocasiones, en busca de una amiloidosis sistémica que pudiera presentarse de forma secundaria ${ }^{10}$.

B I B L I O G R A F Í A

1. Jiménez Cruz JF, Rioja Sanz LA. En: Tomo I, editor. Otras cistopatías. Tratado de Urología. Barcelona: J.R. Prous Editores; 1993:655.

2. González Naranjo F, Velasco Alonso J, Méndez López M, Escaf Barmadah S, González Tuero JS, Capdevila Hernández J. Bladder amyloidosis. Actas Urol Esp. 1992;16:579-81.

3. Caballero Giné JM, Borrat Font P, Martí Picas L, Ristol Pont J. Amiloidosis vesical: Presentación de dos casos. Actas Urol Esp. 2000;24:413-5.

4. Nurmi MJ, Ekfors TO, Puntala PV. Secondary amyloidosis of the bladder: a cause of massive hematuria. J Urol. 1987;138: 44-5.

5. Tesedo Cubero J, Ristol RM, Fernández Zuazu J, Dinares J, Autanell J. Massive hematuria and bladder perforation, complication of systemic amyloidosis. Arch Esp Urol. 1991;44:862-4.

6. Begara Morillas F, Silmí Moyano A, Espinosa Fernández B, Prieto Chiparro L, Rapariz González M, González Morales ML, et al. Bladder amyloidosis in a female patient with suspected bladder carcinoma. Arch Esp Urol. 1994;47:176-9.

7. Caldamone AA, Elbadawi A, Moshtagi A, Frank IN. Primary localizad amyloidosis of urinary bladder. Urology. 1980;15: 174-180.

8. Linke RP. Highly sensitive diagnosis of amyloid and various amyloid syndromes using Congo red fluorescence. Virchows Arch. 2000;436:439-48.

9. Nurmi MJ, Ekfors TO, Rajala PO, Puntala PV. Intravesical dimethyl sulfoxide instillations in the teratment of secondary amyloidosis of the bladder. J Urol. 1990;143:808-10.

10. Malek RS, Wahner-Roedler DL, Gertz MA, Kyle RA. Primary localized amyloidosis of the bladder: experience with dimethyl sulfoxide therapy. J Urol. 2002;168:1018-20.

N. Senarriaga Ruiz de la Illa*, A. Loizaga Iriarte, I. Lacasa Viscasillas y M. Unda Urzaiz

Servicio de Urología, Hospital de Basurto, Universidad del País Vasco, Bilbao, España

*Autor para correspondencia.

Correo electrónico: nerea.senarriagaruiz@osakidetza.net (N. Senarriaga Ruiz de la Illa).

\section{Construcción del ombligo en la extrofia vesical}

\section{Umbilical reconstruction in patients with vesical extrofia}

\section{Sr. Director:}

La ausencia de ombligo tras la cirugía reconstructiva de la extrofia vesical constituye un defecto que agrava, el ya normalmente pobre aspecto estético de la pared abdominal de estos pacientes. La técnica de la reconstrucción umbilical «tipo canguro» ${ }^{1}$, realizada mediante un colgajo cutáneo de base inferior, constituye una técnica sencilla y eficaz, que 